\title{
Free and Total Dopamine in Human Plasma: Effects of Posture, Age and Some Pathophysiological Conditions
}

\author{
Kiyoko Hashizume, Atsushi Yamatodani* , and Toshio Ogihara**
}

\begin{abstract}
Although dopamine (DA) has an important role also outside the central nervous system, the physiological significance of DA in the peripheral plasma is not clearly known. In the present study, we assayed the levels of free and total (free plus conjugated) DA in the human plasma to examine the effect of posture, age and some pathophysiological conditions. Nine healthy volunteers were subjected for head up tilt study. For the examination of the effect of age and some pathophysiological conditions, venous blood samples were taken after overnight fast in 64 control subjects who have no obvious disease and patients who had essential hypertension or congestive heart failure (CHF). The plasma free adrenaline (AD), noradrenaline (NA) and DA were determined by a HPLC-diphenylethylenediamine method and total DA was determined by the same HPLC method after acid hydrolysis. After tilting neither free nor total DA changed significantly despite the increased plasma AD and NA levels. Plasma free NA, DA and total DA levels were correlated positively with age in control subjects but not in the hypertensive nor CHF patients. Patients with CHF showed higher levels of plasma free AD, NA and DA and freeDA/totalDA ratio than did in control subjects. We suspect that plasma DA increased with age partially due to change in renal catecholamine handling and cardiac function. (Hypertens Res 1995; 18 Suppl. I: S205S207)
\end{abstract}

Key Words: plasma dopamine, HPLC, tilting, aging, congestive heart failure

Although dopamine (DA) has an important role outside the central nervous system $(1,2)$ the origin and physiological activities of DA in the plasma is not clearly known. Circulating DA presents almost entirely (more than 99\%) in the conjugated form as sulfate esters in the dogs, monkeys and humans. There are few reports on the plasma free DA level, probably mainly because no sensitive method for assay of plasma free DA is available. In the present study, we assayed the levels of free and total DA in the human plasma to examine the effect of posture, age and some pathophysiological conditions.

\section{Methods}

\section{Subjects}

Nine healthy and overnight-fasted volunteers, 7 men and 2 women, mean age $33.8 \pm 2.6$ years (27-51 years) were subjected $70^{\circ}$ head up tilt study for $10 \mathrm{~min}$ after $10 \mathrm{~min}$ supine position. Blood pressure, heart rate and blood samples were taken at $0 \mathrm{~min}$ and $10 \mathrm{~min}$ of tilting. Sixty-four control subjects, 25 men and 38 women, mean age $61.0 \pm$ 2.8 years (18-96 years) who had a normal blood pressure and no detectable systemic diseases and patients who had essential hypertension or congestive heart failure (CHF) caused by ischemic heart diseases or valvular diseases were studied. Institutionalized elderlies who have no obvious disease were included in the control subjects. All subjects were free of severe renal disease. Venous blood samples were taken at supine position on the morning after an overnight fast. The study was approved by the institutional ethical committee and the subjects gave informed consent.

\section{DA Assay}

Blood samples $(2 \mathrm{ml})$ were drawn into an ice-cold tube containing $20 \mu \mathrm{l}$ of $0.2 \mathrm{M}$ EDTA2Na and $20 \mu \mathrm{l}$ of $0.2 \mathrm{M} \mathrm{Na}_{2} \mathrm{~S}_{2} \mathrm{O}_{5}$ and the plasma was promptly separated by centrifugation at 4,000 rpm for $10 \mathrm{~min}$ at $4^{\circ} \mathrm{C}$. Then the plasma was mixed with a half volume of $6 \%$ perchloric acid and stored at $-80^{\circ} \mathrm{C}$ until assay. Before use, the frozen samples were centrifuged at $13,000 \mathrm{rpm}$ for $30 \mathrm{~min}$ at $4^{\circ} \mathrm{C}$ and the deproteinized supernatant was separated and free adrenaline (AD), NA and DA were determined by HPLC-diphenylethylenediamine method (HLC$725 \mathrm{CA}$, TOSOH, Tokyo). Total DA was determined by the same HPLC method after acid hy-

From the Department of Internal Medicine, Hanwa-Senboku Hospital, *Department of Medical Physics, School of Allied Health Sciences, Faculty of Medicine, Osaka University, ${ }^{* *}$ Department of Geriatric Medicine, Osaka University Medical School, Suita, Japan.

Address for Reprints: Kiyoko Hashizume, M.D., Department of Internal Medicine, Hanwa-Senboku Hospital, 3176 Fukaikita-machi, Sakai 593, Japan. 

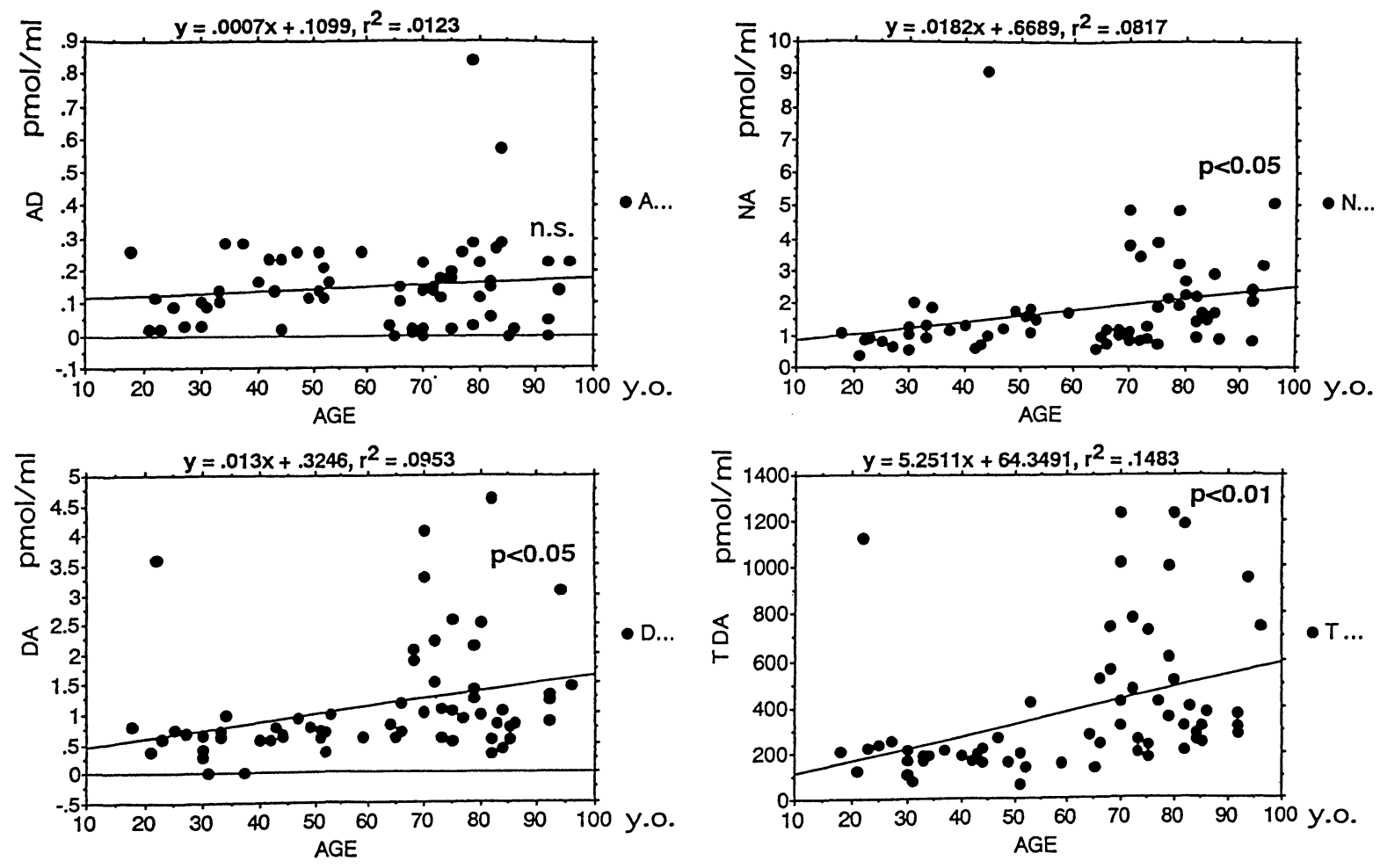

Fig. 1. Correlation between age and catecholamines in control subjects.

Table 1. Plasma Catecholamine Levels in CHF Patients and Age Matched Control Subjects

\begin{tabular}{lccc}
\hline & CHF patients $(n=15)$ & Control subjects $(n=37)$ & $p$-Value \\
\hline Age (y.o.) & $80.4 \pm 9.6$ & $77.7 \pm 1.5$ & $\mathrm{n} . \mathrm{s}$. \\
Free AD (pmol/ml) & $0.67 \pm 0.42$ & $0.17 \pm 0.03$ & $<0.01$ \\
Free NA (pmol/ml) & $3.53 \pm 0.92$ & $2.00 \pm 0.21$ & $<0.05$ \\
Free DA (pmol/ml) & $2.69 \pm 0.69$ & $1.43 \pm 0.17$ & $<0.02$ \\
Total DA (pmol/ml) & $660.7 \pm 144.3$ & $504.1 \pm 53.2$ & $\mathrm{n} . \mathrm{s}$. \\
Free/total DA $(\%)$ & $0.39 \pm 0.05$ & $0.29 \pm 0.01$ & $<0.01$ \\
\hline
\end{tabular}

Values are mean \pm SEM.

drolysis for $10 \mathrm{~min}$ at $120^{\circ} \mathrm{C}$. Differences between values before and after tilting were evaluated by the paired $t$ test. Relationships of age and plasma catecholamines and of plasma NA and other catecholamines were determined by linear regression analysis. The differences between control groups and patients with essential hypertension or $\mathrm{CHF}$ were evaluated by the unpaired $t$ test.

\section{Results}

After head up tilting, mean blood pressure did not change but heart rate increased significantly. Despite the increased plasma $\mathrm{AD}(0.13 \pm 0.029$ $\mathrm{pmol} / \mathrm{ml} v$ s. $0.22 \pm 0.047 \mathrm{pmol} / \mathrm{ml}, p<0.05)$ and NA $(1.08 \pm 0.16 \mathrm{pmol} / \mathrm{ml}$ vs. $2.02 \pm 0.25 \mathrm{pmol} / \mathrm{ml}, p<$ $0.01)$ after head up tilting, neither free DA $(0.43 \pm$ $0.094 \mathrm{pmol} / \mathrm{ml} v s$. $0.45 \pm 0.093 \mathrm{pmol} / \mathrm{ml})$ nor total DA $(174.6 \pm 18.2 \mathrm{pmol} / \mathrm{ml} v s .184 .5 \pm 12.5 \mathrm{pmol} / \mathrm{ml})$ changed significantly. Plasma free NA, DA and tot- al DA levels were correlated positively with age in control subjects (Fig. 1) but not in the hypertensive patients or CHF patients. Plasma AD and total DA levels were correlated positively with plasma NA levels but no correlation was observed between plasma free DA and NA. Patients who had CHF were higher plasma free $\mathrm{AD}, \mathrm{NA}$ and $\mathrm{DA}$ levels and free DA/total DA ratio than age matched control subjects (Table 1). Free and total DA levels did not correlate with plasma NA levels in CHF patients. In the hypertensive patients plasma free and total DA levels showed little difference from control subjects.

\section{Discussion}

Head up tilting elicits a neurohormonal response characterized by increases in heart rate and circulating NA levels. But plasma free DA and total DA did not increase in response to upright tilt. So 
changes in plasma DA may not be parallel to the sympathetic tone. Although it is well known that plasma NA levels showed significant age related elevation (3), there are few reports which examined effect of age on plasma DA and it is still controversial. It is reported that dopaminergic activity decreased in advanced age (4). In our study, elderly persons have large inter-individual variation and some have considerably high plasma DA levels. We have observed patients with chronic renal failure taking hemodialysis therapy had marked high DA conjugate levels (unpublished). We also reported that one of DA conjugates isomer, 3-O-sulphate levels in plasma were correlated positively with serum creatinine levels (5). Thus, it is suspected that renal catecholamine handling may contribute to the high plasma DA levels. We found that CHF patients showed significant higher free DA levels compared with age matched controls but the reason is not clear. In conclusion we suggest that plasma free and total DA increase with age partially due to changes in renal catecholamine handling or/and cardiac function.

\section{References}

1. Toda N, Goldberg LI: Effects of dopamine on isolated canine coronary arteries. Cardiovasc Res 1975; 9: 384-389.

2. Lee MR: Dopamine and the kidney. Clin Sci 1982; 62: 439-448.

3. Masuo K, Ogihara T, Kumahara Y, Yamatodani A, Wada H: Age-dependence of hypertensive-normotensive differences in plasma norepinephrine under three sodium intakes. Folia Endocrinol Jpn 1983; 59: 12761285.

4. Kuchel OG, Kuchel GA: Peripheral dopamine in pathophysiology of hypertension: interaction with aging and lifestyle. Hypertension 1991; 18: 709-721.

5. Hashizume K, Ogihara T, Yamatodani A, Yamamoto T, Wada H, Kumahara Y: Plasma levels and renal clearance of two isomers of dopamine sulphate in patients with essential hypertension. Clin Exp Hypertens 1988; A10: 561-574. 